\title{
Design of Computer Experiments for Optimization, Estimation of Function Contours, and Related Objectives
}

\author{
Derek Bingham, Pritam Ranjan, and William J. Welch \\ Simon Fraser University, Acadia University, and University of British Columbia
}

\section{Introduction}

A computer code or simulator is a mathematical representation of a physical system, for example a set of differential equations. Such simulators take a set of input values or conditions, $\mathbf{x}$, and from them produce an output value, $y(\mathbf{x})$, or several such outputs. For instance, one application we use for illustration simulates the average tidal power, $y$, generated as a function of a turbine location, $\mathbf{x}=\left(x_{1}, x_{2}\right)$, in the Bay of Fundy, Nova Scotia, Canada (Ranjan et al. 2011). Performing scientific or engineering experiments via such a computer code is often more time and cost effective than running a physical experiment or collecting data directly.

A computer experiment often has similar objectives to a physical experiment. For example, computer experiments are often used in manufacturing or process development. If $y$ is a quality measure for a product or process, an experiment could aim to optimize $y$ with respect to $\mathbf{x}$. Similarly, an experiment might aim to find sets or contours of $\mathbf{x}$ values that make $y$ equal a specified target value. Such scientific and engineering objectives are naturally and efficiently achieved via so-called data-adaptive sequential design, which we describe below. Essentially, each new run (that is, new set of input values) is chosen based on analysis of the data so far, to make the best expected improvement in the objective. In a computer experiment, choosing new experimental runs, re-starting the experiment, etc. pose only minor logistical challenges if these decisions are also computer-controlled, a distinct advantage relative to a physical experiment.

Choosing new runs sequentially for optimization, moving $y$ to a target, etc. has been formalized using the concept of expected improvement (Jones et al. 1998). The next experimental run is made where the expected improvement in the function of interest is largest. This expectation is with respect to the predictive distribution of $y$ from a statistical model relating $y$ to $\mathbf{x}$. By considering a set of possible inputs $\mathbf{x}$ for the new run, we can choose that which gives the largest expectation.

We illustrate this basic idea with two examples in Section 2. Then we describe formulations of 
improvement functions and their expectations in Section 3, Expectation implies a statistical model, and in Section 4 we outline the use of Gaussian process models for fast emulation of computer codes. In Section 5 we describe some extensions to other, more complex scientific objectives.

\section{Expected improvement and sequential design: basic ideas}

We illustrate the basic idea of expected improvement and data-adaptive sequential design via two examples. The first, a tidal-power application, shows the use of expected improvement in sequential optimization. We then use a simulator of volcanic pyroclastic flow to illustrate how to map out a contour of a function.

\subsection{Optimization}

Ranjan et al. (2011) described output from a 2D computer-model simulation of the power produced by a tidal turbine in the Minas Passage of the Bay of Fundy, Nova Scotia, Canada. In this simplified version of the problem there are just two inputs for the location of a turbine. Originally, the input space was defined by latitude-longitude coordinates for a rectangular region in the Minas Passage (see Figure 5 of Ranjan et al. 2011). The coordinates were transformed so that $x_{1}$ is in the direction of the flow and $x_{2}$ is perpendicular to the flow. Furthermore, only an interesting part of the Minas Passage was considered, with $x_{1} \in[0.75,0.95]$ and $x_{2} \in[0.2,0.8]$. The code generates $y$, the extractable power in MW, averaged over a tidal cycle.

For the simplified demonstration here, $y$ was computed for 533 runs on a $13 \times 41$ grid of $x_{1}$ and $x_{2}$ values, which produced the contour plot of Figure 1(a).

We now demonstrate how the turbine location optimizing the power, i.e., $\max y\left(x_{1}, x_{2}\right)$, can be found with far fewer than 533 runs of the computer code. Such an approach would be essential for the computer experiment of ultimate interest. A more realistic computer model has a grid resolution 10 times finer in each coordinate and introduces vertical layers in a 3D code. The running time would be increased by several orders of magnitude. Moreover, the final aim is to position several turbines, which would interfere with each other, and so the optimization space is larger than two or three dimensions. Thus, the ultimate goal is to optimize a high-dimensional function with a limited number of expensive computer model runs. Inevitably, much of the input space cannot be explicitly explored, and a statistical approach to predict outcomes (extractable power) along with an uncertainty measure is required to decide where to make runs and when to stop. The expected improvement criterion addresses these two requirements.

Thus, imagine a more limited computer experiment with just 20 runs, as shown by the points in Figure 1, The experimental design (that is, the locations of the 20 points) is a maximin Latin hypercube (Morris \& Mitchell 1995), a stratified scheme that is "space-filling" even in higher dimensions. The choice of 20 runs is based on the heuristic rule that an initial computer experiment 

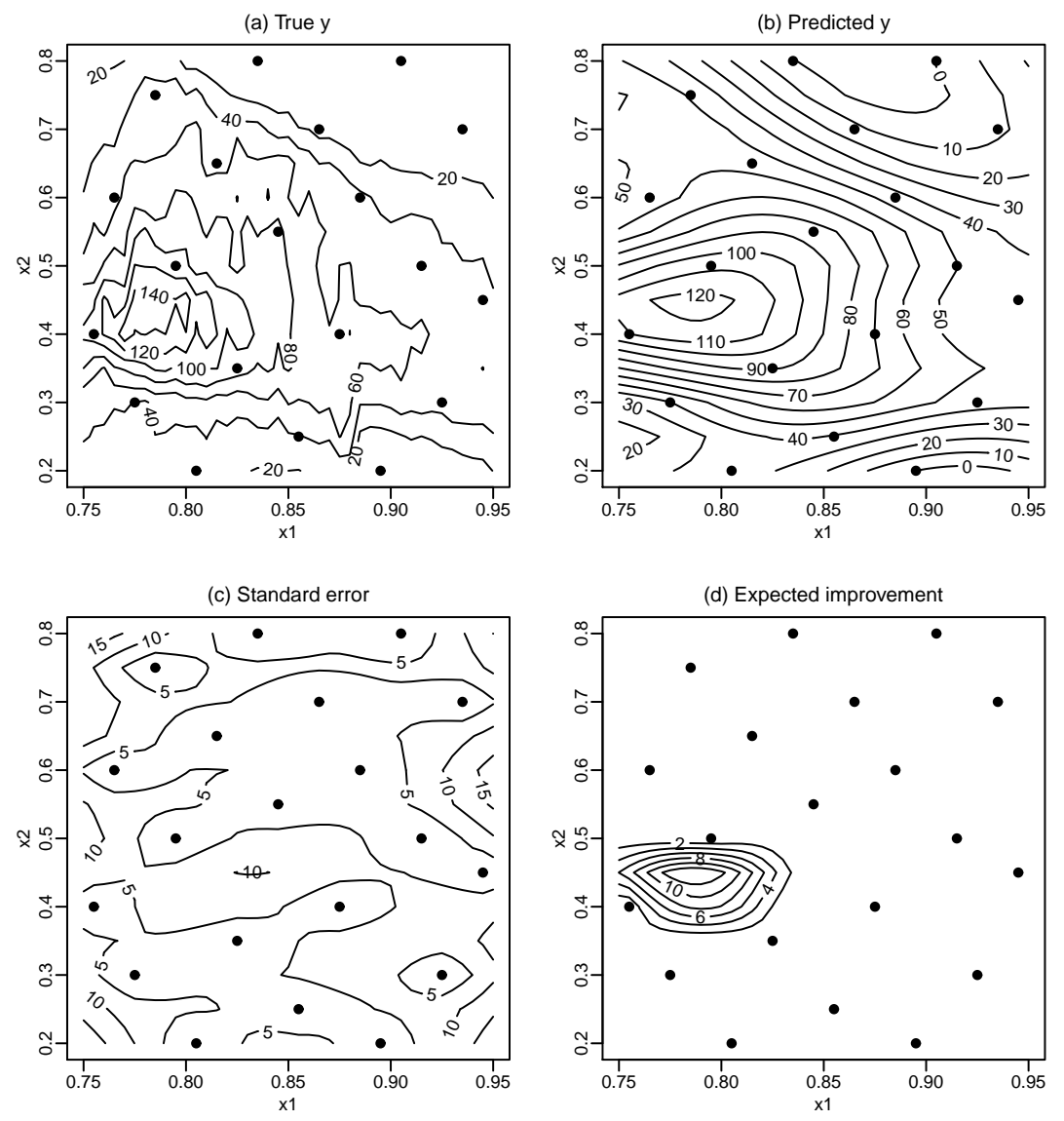

Figure 1: Initial 20-run design and analysis for the tidal-power application: (a) true power, $y$, in MW; (b) predicted power, $\hat{y}(\mathbf{x})$; (c) standard error, $s(\mathbf{x})$; and (d) expected improvement, $E[I(\mathbf{x})]$. The design points from an initial 20-run maximin Latin hypercube are shown as filled circles. All plots are functions of the two input variables, $x_{1}$ and $x_{2}$, which are transformations of longitude and latitude. 
has $n=10 d$ observations (Loeppky et al. 2009), where $d$ is the input dimension; here $d=2$. Among the 20 initial runs, the largest $y$ observed, denoted by $y_{\max }^{(20)}$, is $109.7 \mathrm{MW}$ at $\left(x_{1}, x_{2}\right)=(0.755,0.4)$. The expected improvement algorithm tries to improve on the best value found so far as new runs are added.

At each iteration of the computer experiment we obtain a predictive distribution for $y(\mathbf{x})$ conditional on the runs so far. This allows prediction of the function at input vectors $\mathbf{x}$ where the code has not been run. A Gaussian process (GP) statistical model is commonly used for prediction, as outlined in Section 4, though this is not essential. A GP model was fit here to the data from the first 20 runs, giving the point-wise predictions, $\hat{y}(\mathbf{x})$, of $y(\mathbf{x})$ in Figure $1(\mathrm{~b})$ and the standard error, $s(\mathbf{x})$, in Figure 1(c). The standard error is a statistical measure of uncertainty concerning the closeness of the predicted value to the actual true value of $\mathrm{y}(\mathrm{x})$. We show the predicted values and standard errors through contours in Figures 1(b) and 1(c).

Figures 1(b) and (c) are informative about regions in the input space that are promising versus unpromising for further runs of the code. While the $\hat{y}(\mathbf{x})$ prediction surface is nonlinear, it suggests there is a single, global optimum. Moreover, the $s(\mathbf{x})$ surface is uniformly below about 15: For much of the input space, $\hat{y}(\mathbf{x})$ is so much smaller than $y_{\max }^{(20)}$ relative to $s(\mathbf{x})$ that a new run is expected to make virtually zero improvement.

The expected improvement (EI) for a candidate new run at any $\mathbf{x}$ is computed from the predictive distribution of $y(\mathbf{x})$. (See Section 3.1 for the formal definition of EI.) Figure 1(d) shows the EI surface based on predictive distributions from a GP that is fitted to the data from the initial 20 runs of the tidal-power code. By the definition in Section 3.1, improvement can never be negative (if the output from the new run does beat the current optimum, the current optimum stands). Thus, EI is always non-negative too. Figure 1(d) indicates that for most of the input space EI is near zero and a new run would be wasted, but there is a sub-region where EI is more than $12 \mathrm{MW}$. Evaluating EI over the $13 \times 41$ grid shows that the maximum EI is 13.9 at $\mathbf{x}=(0.785,0.45)$. In other words, a new code run to evaluate $y(0.785,0.45)$ is expected to beat $y_{\max }^{(20)}=109.7$ by about 14.

Thus, run 21 of the sequential design for the computer experiment is at $\mathbf{x}^{(21)}=(0.785,0.45)$. The actual power obtained from the simulator is $y=159.7 \mathrm{MW}$, so the best $y$ found after 21 runs is $y_{\max }^{(21)}=159.7$, and this is the value to beat at the next iteration. Note that the actual improvement in the optimum from the new run is $159.7-109.7=50.0$, compared with an expectation of about 13.9 .

The new run raises concerns about the statistical model. Before making the new run, the predictive distribution of $y\left(\mathbf{x}^{(21)}\right)$ is approximately normal, $N\left(123.5,5.67^{2}\right)$, an implausible distribution given the large value of the standardized residual $\left(y\left(\mathbf{x}^{(21)}\right)-\hat{y}\left(\mathbf{x}^{(21)}\right)\right) / s\left(\mathbf{x}^{(21)}\right)=(159.7-$ $123.5) / 5.67=6.4$. One deficiency is that $s(\mathbf{x})$ may not reflect all sources of uncertainty in estimation of the parameters of the GP (see Section 44). A more important reason here, however, 

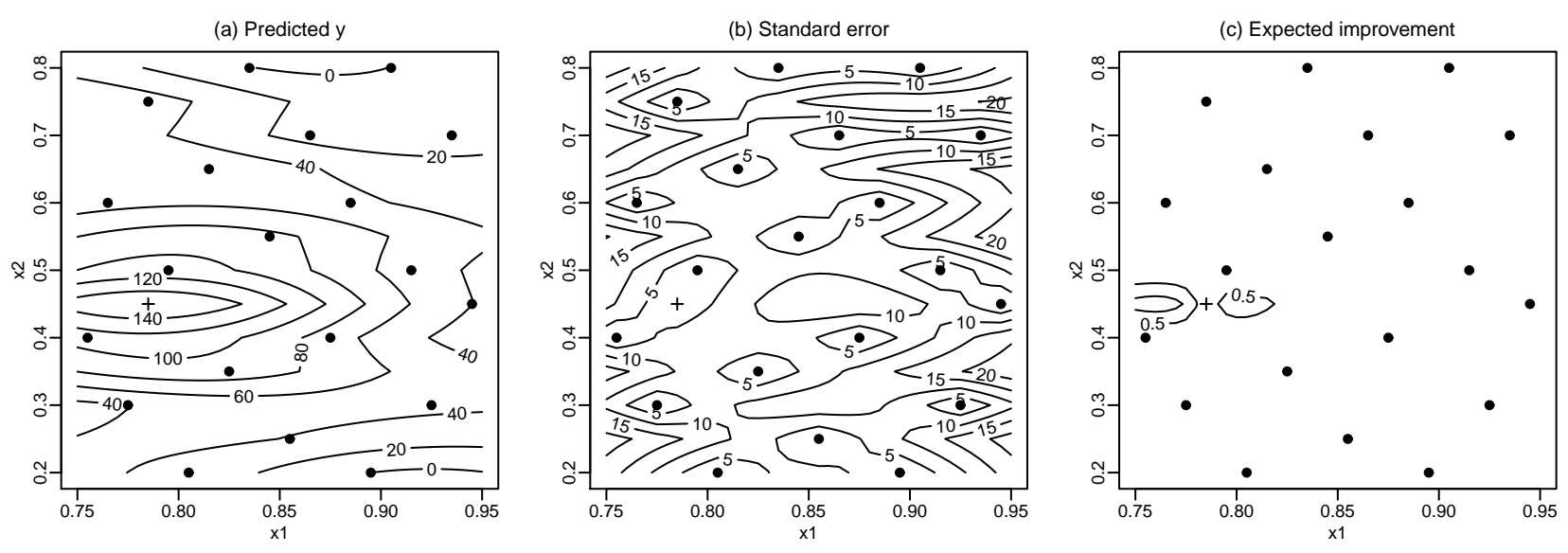

Figure 2: Analysis of the tidal-power application after 21 runs: (a) predicted power, $\hat{y}$; (b) standard error, $s(\mathbf{x})$; and (c) expected improvement, $I(\mathbf{x})]$. The new design point is shown as a " + ".

is that the new observation successfully finds a peak in the input space, a sub-region where the output function is growing rapidly and uncertainty is larger. In contrast, the first 20 runs were at locations where the function is flatter and easier to model. The GP model fit to the initial runs under-estimated the uncertainty of prediction in a more difficult part of the input space.

Careful consideration of the properties of a GP model and the possible need for transformations is particularly relevant for sequential methods based on predictive distributions. Uncertainty of prediction is a key component of the EI methodology, so checking that a model has plausible standard errors of prediction is critical.

One way of improving the statistical emulator of the tidal-power code is to consider transformation of the output. This is described in the context of the volcano example of Section 2.2, where transformation is essential. For the tidal-power example, persisting with the original model will show that it adapts to give more plausible standard errors with a few more runs.

The GP model and predictive distributions are next updated to use the data from all 21 runs now available. Figure 2(a) shows the location of the new run as a "+" and the updated $\hat{y}(\mathbf{x})$. Similarly, Figure 2(b) gives the updated $s(\mathbf{x})$. A property of the GP fit is that $s(\mathbf{x})$ must be zero at any point $\mathbf{x}$ where $y(\mathbf{x})$ is in the data set for the fit (see Jones et al. (1998) for a derivation of this result). Thus, $s(\mathbf{x})$ is zero at the new run, and Figure 2(b) shows it is less than 5 near the new run. Comparing with Figure 1(c), it is seen that $s(\mathbf{x})$ was 5 or more in this neighbourhood for the GP fit before the new run.

On the other hand, comparison of Figures 1(c) and 2(b) shows that $s(\mathbf{x})$ has increased outside the neighbourhood of the new run. For example, at the right edge of Figure 1(c), s(x) barely reaches 15 , yet $s(\mathbf{x})$ often exceeds 15 or even 20 at the same locations in Figure 2(b). The 21-run GP fit has adapted to reflect the observed greater sensitivity of the output to $x_{1}$ and $x_{2}$. (For instance, the estimate of the GP variance parameter $\sigma^{2}$, defined in Section 4 , increases.) Thus, the 
model has at least partially self corrected and we continue with it.

The EI contour plot in Figure 2(c) suggests that there is little further improvement to be had from a further run anywhere. If a run number 22 is made, however, it is not located where $\hat{y}(\mathbf{x})$ is maximized; that location coincides with run 21 and there would be no gain. Rather, the maximum EI of about 1.3 MW occurs at a moderate distance from the location providing maximum $\hat{y}(\mathbf{x})$. As we move away from run 21, the standard error increases from zero until it is large enough to allow a modest expected improvement. Thus, this iteration illustrates that EI trades off local search (evaluate where $\hat{y}(\mathbf{x})$ is optimized) and global search (evaluate where uncertainty concerning fitted versus actual output values, characterized by $s(\mathbf{x})$, is optimized).

With this approach, EI typically indicates smaller potential gains as the number of iterations increases. Eventually, the best EI is deemed small enough to stop. It turns out that run 21 found the global maximum for extractable power on the $13 \times 41$ grid of locations.

\subsection{Contour estimation}

We illustrate sequential design for mapping out a contour of a computer-model function using TITAN2D computer model runs provided by Elaine Spiller. They relate to the Colima volcano in Mexico. Again for ease of illustration, there are two input variables: $x_{1}$ is the pyroclastic flow volume $\left(\mathrm{m}^{3}\right)$ of fluidized gas and rock fragments from the eruption; and $x_{2}$ is the basal friction angle in degrees, defined as the the minimum slope for the volcanic material to slide. The output $z$ is the maximum flow height $(\mathrm{m})$ at a single, critical location. As is often the case, the code produces functional output, here flow heights over a 2D grid on the earth's surface, but the output for each run is reduced to a scalar quantity of interest, the height at the critical location.

Following Bavarri et al. (2009), the scientific objective is to find the values of $x_{1}$ and $x_{2}$ where $z=1$, a contour delimiting a "catastrophic" region. Bayarri et al. (2009) used the same TITAN2D code but for a different volcano. They also conducted their sequential experiment in a less formal way than in our illustration of the use of EI.

There are 32 initial runs of the TITAN2D code. They are located at the points shown in Figure 3(a). The predicted flow height surface also shown in Figure 3(a) relates to a GP model fit to the transformed simulator output $y=\sqrt{z}$. This choice was made by trying GP models on three different scales: the $z$ untransformed height; $\log (z+1)$, as chosen by Bayarri et al. (2009); and $\sqrt{z}$. Our final choice of $y=\sqrt{z}$ results from inspection of standard cross-validation diagnostics for GP models (Jones et al. 1998).

The dashed curve in Figure 3 (a) shows the contour where $\hat{y}(\mathbf{x})=1$. This maps out the contour of interest in the $\left(x_{1}, x_{2}\right)$ input space, but it is based on predictions subject to error. The standard errors in Figure 3(b) are substantial, and sequential design via EI aims to improve the accuracy of the estimate of the true $y(\mathbf{x})=1$ contour. 

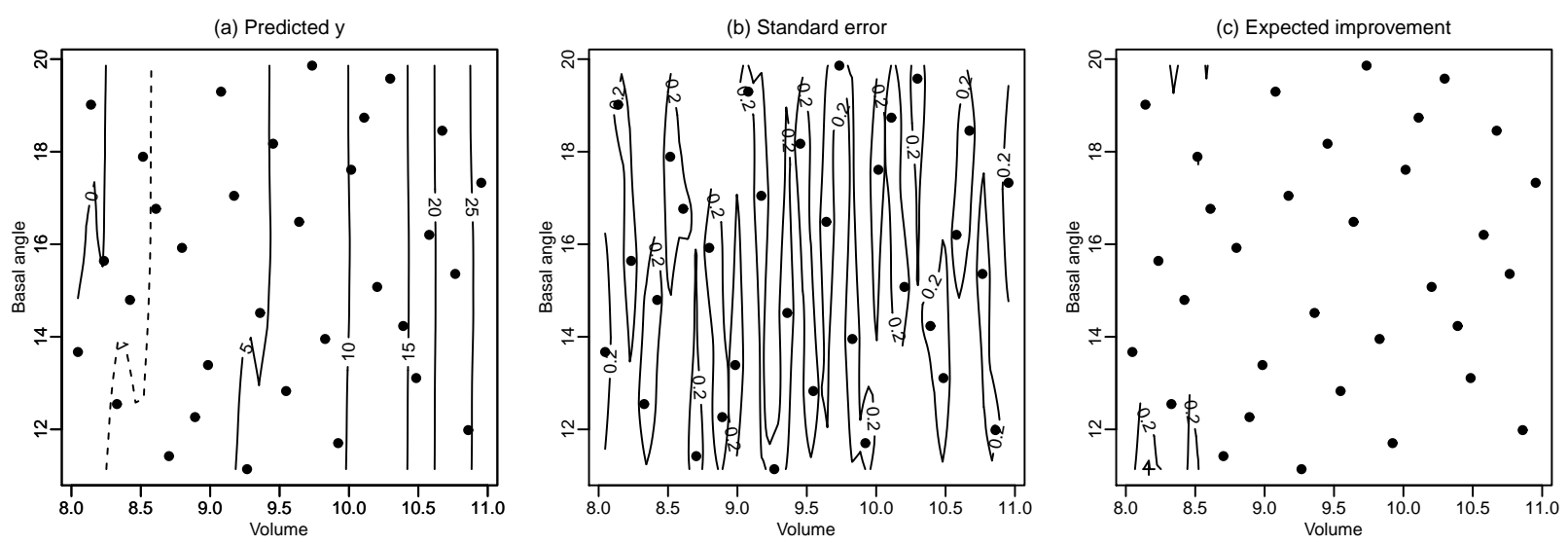

Figure 3: Analysis of the initial 32-run design for the volcano application: (a) predicted height, $\hat{y}(\mathbf{x})$, where $y=\sqrt{z}$; (b) standard error, $s(\mathbf{x})$; and (c) expected improvement, $E[I(\mathbf{x})]$. The design points of the initial 32-run design are shown as filled circles. The new design point chosen by the EI criterion is shown as a "+" in the lower left corner of (c).

The EI criterion adapted for the contouring objective is defined in Section 3.2, It is computed for the initial design of the volcano example in Figure 3(c). EI suggests improving the accuracy of the contour by taking the next run at $\left(x_{1}, x_{2}\right)=(8.2,11.1)$. Inspection of Figures 3 (a) and 3(b) show that this location is intuitively reasonable. It is in the vicinity of the predicted $\hat{y}(\mathbf{x})=1$ contour and has a relatively large predictive standard error. Reducing substantial uncertainty in the vicinity of the estimated contour is the dominant aspect of the EI measure of equation (3).

The tidal-flow and volcano applications both have a 2D input space for ease of exposition, but the same approaches apply to higher dimensions, where choosing runs in a sequential design would be more problematic with ad hoc methods.

\section{$3 \quad$ Expected Improvement Criteria}

In this section, we briefly define improvement and EI in general. We then review two implementations, specific to global optimization and contour estimation, respectively.

Let $I(\mathbf{x})$ be an improvement function defined for any $\mathbf{x}$ in the input space, $\chi$. It depends on the scientific objective, such as improving the largest $y$ found so far in maximization. In general, it is formulated for efficient estimation of a pre-specified computer-model feature, $\psi(y)$. Typically, before taking another run, $I(\mathbf{x})$ is an unobserved function of $\mathbf{x}$, the unknown computer-model output $y(\mathbf{x})$, the predictive distribution of $y(\mathbf{x})$, and the best estimate so far of $\psi(y)$.

Given a definition of $I(\mathbf{x})$, as its name suggests the corresponding EI criterion is given by the expectation of $I(\mathbf{x})$ :

$$
E[I(\mathbf{x})]=\int I(\mathbf{x}) f(y \mid \mathbf{x}) d y
$$


Here expectation is with respect to $f(y \mid \mathbf{x})$, the predictive distribution of $y(\mathbf{x})$ conditional on all runs so far. Assuming the sequential design scheme selects one new input point at a time, the location of the new point, $\mathbf{x}^{\text {new }}$, is the global maximizer of $E[I(\mathbf{x})]$ over $\mathbf{x} \in \chi$.

\subsection{EI for global optimization}

Finding the global minimum, $\psi(y)=\min \{y(\mathbf{x}): x \in \chi\}$, of an expensive to evaluate function is an extensively investigated optimization problem. (Finding the maximum is reformulated as $\min -y(\mathbf{x})$, and the following results apply.) Jones et al. (1998) proposed an efficient sequential solution via the improvement function to assess the gain if a new evaluation is made at $\mathbf{x}$. The improvement function is

$$
I(\mathbf{x})=\max \left\{y_{\min }^{(n)}-y(\mathbf{x}), 0\right\}
$$

where $y_{\min }^{(n)}$ is the minimum value of $y$ found so far with $n$ runs. The objective is improved by $y_{\min }^{(n)}-y(\mathbf{x})$ if $y_{\min }^{(n)}>y(\mathbf{x})$, otherwise there is no improvement.

The GP statistical model outlined in Section 4 leads to a Gaussian predictive distribution for $f(y \mid \mathbf{x})$, i.e., $y(\mathbf{x}) \sim N\left(\hat{y}(\mathbf{x}), s^{2}(\mathbf{x})\right)$. The Gaussian predictive model leads to a simple, closed form for the expected improvement:

$$
E[I(\mathbf{x})]=s(\mathbf{x}) \phi(u)+\left(y_{\min }^{(n)}-\hat{y}(\mathbf{x})\right) \Phi(u)
$$

where $u=\left(y_{\min }^{(n)}-\hat{y}(\mathbf{x})\right) / s(\mathbf{x})$, and $\phi(\cdot)$ and $\Phi(\cdot)$ denote the standard normal probability density function (pdf) and cumulative distribution function (cdf), respectively.

Large values of the first term support global exploration in regions of the input space sparsely sampled so far, where $s(\mathbf{x})$ is large. The second term favours search where $\hat{y}(\mathbf{x})$ is small, which is often close to the location giving $y_{\min }^{(n)}$, i.e., local search. This trade-off between local and global search makes EI-based sequential design very efficient, and it often requires relatively few computermodel evaluations to achieve a desired accuracy in estimating min $y$.

For instance, in the tidal-power application, the EI surface in Figure 1(d)) indicates that the first follow-up run is at the location giving the maximum predicted power (see Figure 2(a)). Thus, the local-search component dominates. Conversely, the suggested location for the second follow-up run is in an unsampled region near the maximum predicted power (see Figure 2(c)).

Attempts have been made to control this local versus global trade-off for faster convergence (that is, using as few runs as possible) to the true global minimum. For instance, Schonlau et al. (1998) proposed an exponentiated improvement function, $I^{g}(\mathbf{x})$, for $g \geq 1$. With $g>1$, there is more weight on larger improvements when expectation is taken to compute EI. Such large improvements will have a non-trivial probability even if $\hat{y}(\mathbf{x})$ is unfavourable, provided $s(\mathbf{x})$ is sufficiently large. Hence, global exploration of high-uncertainty regions can receive more attention with this adaptation. Similarly, Sóbester et al. (2005) developed a weighted expected improvement function 
(WEIF) by introducing a user-defined weight parameter $w \in[0,1]$ in the Jones et al. (1998) EI criterion, and Ponweiser et al. (2008) proposed clustered multiple generalized expected improvement.

\subsection{EI for contour estimation}

Ranjan et al. (2008) developed an EI criterion specific to estimating a threshold (or contour) of $y$. They applied it to a 2-queue 1-server computer network simulator that models the average delay in a queue for service.

Let the feature of interest $\psi(y)$ be the set of input vectors $\mathbf{x}$ defining the contour at level $a$ :

$$
S(a)=\{x: y(\mathbf{x})=a\}
$$

The improvement function proposed by Ranjan et al. (2008) is

$$
I(\mathbf{x})=\epsilon^{2}(\mathbf{x})-\min \left\{(y(\mathbf{x})-a)^{2}, \epsilon^{2}(\mathbf{x})\right\},
$$

where $\epsilon(\mathbf{x})=\alpha s(\mathbf{x})$ for a positive constant $\alpha$ (e.g., $\alpha=1.96$, corresponding to $95 \%$ confidence/credibility under approximate normality). This improvement function defines a limited region of interest around $S(a)$ for further experimentation. Point-wise, the extent of the region depends on the uncertainty $s(\mathbf{x})$ and hence the tolerance $\epsilon(\mathbf{x})$.

Under a normal predictive distribution, $y(\mathbf{x}) \sim N\left(\hat{y}(\mathbf{x}), s^{2}(\mathbf{x})\right)$, the expectation of $I(\mathbf{x})$ can again be written in closed form:

$$
\begin{aligned}
E[I(\mathbf{x})] & =\left[\epsilon^{2}(\mathbf{x})-(\hat{y}(\mathbf{x})-a)^{2}\right]\left(\Phi\left(u_{2}\right)-\Phi\left(u_{1}\right)\right) \\
& +s^{2}(\mathbf{x})\left[\left(u_{2} \phi\left(u_{2}\right)-u_{1} \phi\left(u_{1}\right)\right)-\left(\Phi\left(u_{2}\right)-\Phi\left(u_{1}\right)\right)\right] \\
& +2(\hat{y}(\mathbf{x})-a) s(\mathbf{x})\left(\phi\left(u_{2}\right)-\phi\left(u_{1}\right)\right)
\end{aligned}
$$

where $u_{1}=(a-\hat{y}(\mathbf{x})-\epsilon(\mathbf{x})) / s(\mathbf{x})$ and $u_{2}=(a-\hat{y}(\mathbf{x})+\epsilon(\mathbf{x})) / s(\mathbf{x})$. Like EI for optimization, the EI criterion in (3) trades off the twin aims of local search near the predicted contour of interest and global exploration. The first term on the right of (3) recommends an input location with a large $s(\mathbf{x})$ in the vicinity of the predicted contour. When it dominates, the follow-up point is often essentially the maximizer of $\epsilon^{2}(\mathbf{x})-(\hat{y}(\mathbf{x})-a)^{2}$. This consideration led to the new point in Figure 3(c) of the volcano application, for instance. The last term in (3) gives weight to points far away from the predicted contour with large uncertainties. The second term is often dominated by the other two terms in the EI criterion.

The EI criterion in (3) can easily be extended to related aims. For simultaneous estimation of $k$ contours $S\left(a_{1}\right), \ldots, S\left(a_{k}\right)$, with $S(\cdot)$ defined in (2), the improvement function becomes

$$
I(\mathbf{x})=\epsilon^{2}(\mathbf{x})-\min \left\{\left(y(\mathbf{x})-a_{1}\right)^{2}, \ldots,\left(y(\mathbf{x})-a_{k}\right)^{2}, \epsilon^{2}(\mathbf{x})\right\}
$$


and the corresponding EI can also be written in a closed form. When interest centres on the 100p-th percentile, $\nu_{p}$, of the simulator output, Roy (2008) suggested sequential design to estimate $S\left(\nu_{p}\right)$ using the improvement function

$$
I^{g}(\mathbf{x})=\epsilon^{g}(\mathbf{x})-\min \left\{\left(y(\mathbf{x})-\hat{\nu}_{p}\right)^{g}, \epsilon^{g}(\mathbf{x})\right\}
$$

Here the contour of interest changes after every follow-up point when $\hat{\nu}_{p}$ is estimated using Monte Carlo methods. Note that $I^{g}(\mathbf{x})$ for $g=2$ is the improvement function in (3) . Here $a=\hat{\nu}_{p}$ and hence not fixed throughout the sequential procedure. That is, $\hat{\nu}_{p}$ for choosing a run is likely to be different from one run to the next. Bichon et al. (2009) adapted this criterion to estimate the probability of rare events and system failure in reliability-based design optimization.

\section{Gaussian Process Models and Predictive Distributions}

Evaluation of an EI criterion requires the computation of the expectation of $I(\mathbf{x})$ with respect to the predictive distribution of $y(\mathbf{x})$. In principle, any predictive distribution can be used, but for the method to be useful, it should faithfully reflect the data obtained up to the run in question. In practice, treating the data from the computer-model runs as a realization of a GP is nearly ubiquitous in computer experiments. A GP model leads to a Gaussian predictive distribution, which in turn leads to the closed form expressions in (11) and (3) and easy interpretation of the trade off between local and global search.

A GP model is a computationally inexpensive statistical emulator of a computer code. A key feature of many codes is that they are deterministic: re-running the computer model with the same values for all input variables will give the same output values. Such a deterministic function is placed within a statistical framework by considering a given computer-model input-output relationship as the realization of a stochastic process, $Z(\mathbf{x})$, indexed by the input vector. A single realization of the process is non-random, hence the relevance for a deterministic computer code. For a continuous function, the process is usually assumed to be Gaussian, possibly after transformation, as was done for the volcano application.

This GP or Gaussian Stochastic Process (GaSP) paradigm for modelling a computer code dates back to Sacks, Schiller \& Welch (1989), Sacks, Welch, Mitchell \& Wynn (1989), Currin et al. (1991), and O'Hagan (1992). Specifically, the code output function, $y(\mathbf{x})$, is treated as a realization of

$$
Y(\mathbf{x})=\mu(\mathbf{x})+Z(\mathbf{x}),
$$

where $\mu(\mathbf{x})$ is a mean (regression) function in $\mathbf{x}$, and $Z(\mathbf{x})$ is a Gaussian process with mean 0 and variance $\sigma^{2}$.

Crucial to this approach is the assumed correlation structure of $Z(\mathbf{x})$. For two configurations of the $d$-dimensional input vector, $\mathbf{x}=\left(x_{1}, \ldots, x_{d}\right)$ and $\mathbf{x}^{\prime}=\left(x_{1}^{\prime}, \ldots, x_{d}^{\prime}\right)$, the correlation between 
$Z(\mathbf{x})$ and $Z\left(\mathbf{x}^{\prime}\right)$ is denoted by $R\left(\mathbf{x}, \mathbf{x}^{\prime}\right)$. Here, $R(\cdot, \cdot)$ is usually a parametric family of functions, for which there are many choices (e.g., Santner et al. 2003, Section 2.3). The computations for the applications in Section 2 were based on a constant (intercept) regression only and a stationary power-exponential correlation function,

$$
R\left(\mathbf{x}, \mathbf{x}^{\prime}\right)=\exp \left(-\sum_{j=1}^{d} \theta_{j}\left|x_{j}-x_{j}^{\prime}\right|^{p_{j}}\right) .
$$

Here, $\theta_{j}$ (with $\theta_{j} \geq 0$ ) and $p_{j}$ (with $1 \leq p_{j} \leq 2$ ) control the properties of the effect of input variable $j$ on the output. A larger value of $\theta_{j}$ implies greater sensitivity (activity) of $y$ with respect to $x_{j}$, whereas a larger value of $p_{j}$ implies smoother behaviour of $y$ as a function of $x_{j}$.

Under this model the output values from $n$ runs of the code, $Y_{1}, \ldots, Y_{n}$, have a joint multivariate normal distribution. If the parameters in the statistical model-in the mean function, in the correlation function, and $\sigma^{2}$-are treated as known, the predictive distribution of $Y$ at a new $\mathbf{x}$ has a normal distribution: $N\left(\hat{y}(\mathbf{x}), s^{2}(\mathbf{x})\right)$, where $\hat{y}(\mathbf{x})$ is the conditional mean of $Y(\mathbf{x})$ given $Y_{1}, \ldots, Y_{n}$, and $s^{2}(\mathbf{x})$ is the conditional variance. Without assuming normality, $\hat{y}(\mathbf{x})$ can also be interpreted as the best linear unbiased predictor, and $s^{2}(\mathbf{x})$ is the associated mean squared error. In practice, the unknown parameters have to be estimated, usually by maximum likelihood or Bayesian methods. The predictive distribution is then only approximately normal. Moreover, Bayesian estimation of the correlation parameters may be necessary to capture all sources of uncertainty in the predictive distribution.

As mentioned already, neither a GP model nor a normal predictive distribution are essential for sequential design with an EI criterion. For instance, Chipman et al. (2012) used the optimization improvement function of Jones et al. (1998) with Bayesian additive regression trees (BART). Thus, the emulator was a non-parametric ensemble of tree models.

\section{Other EI-based criteria}

Over the last two decades, a plethora of EI-based criteria have been proposed for other scientific and engineering objectives.

Applications can involve several outputs of interest. For instance, constrained optimization problems arise where the code generating the objective function $y(\mathbf{x})$ or another code gives values for a constraint function, $c(\mathbf{x})$, (or several functions). For a feasible solution, $c(\mathbf{x})$ must lie in $[a, b]$. If $c(\mathbf{x})$ is also expensive to compute, one can build an emulator, $\hat{c}(\mathbf{x})$, for it too. The predictive distribution for $c(\mathbf{x})$ leads to an estimate of the probability that $a<c(\mathbf{x})<b$ for any new run $\mathbf{x}$ under consideration. EI in (11) is multiplied by this probability of feasibility to steer the search to locations where EI for the objective $y(\mathbf{x})$ is large and $c(\mathbf{x})$ is likely to be feasible (Schonlau et al. 1998). For a code with multivariate output, Henkenjohann \& Kunert (2007) proposed an EI criterion for 
estimating the global maximum of the desirability scores of simulator outputs.

Lehman et al. (2004) developed improvement functions for finding $M$ - and $V$-robust designs for optimization of an engineering process. Here the simulator inputs include both controllable and environmental (uncontrollable noise) variables. ("Uncontrollable" here means in the field; all inputs are typically set at specified values in a simulator run.) For a given configuration of the control variables, $\mathbf{x}_{c}$, let $\mu\left(\mathbf{x}_{c}\right)$ and $\sigma^{2}\left(\mathbf{x}_{c}\right)$ be the unknown mean and variance of the simulator output $y$ with respect to the distribution of the environmental variables. An $M$-robust engineering design minimizes $\mu\left(\mathbf{x}_{c}\right)$ with respect to $\mathbf{x}_{c}$ subject to a constraint on $\sigma^{2}\left(x_{c}\right)$, whereas $V$-robust engineering design minimizes $\sigma^{2}\left(x_{c}\right)$ subject to a constraint on $\mu\left(x_{c}\right)$.

The inclusion of measurement error (or equivalently, considering a nondeterministic simulator) is becoming more popular in computer experiments, often due to unavoidable simulator biases and inaccurate modelling assumptions. Minimizing a noisy mean output response is perhaps undesirable, and Ranjan (2013) recommended minimizing a lower quantile, $q(\mathbf{x})$, via an estimate $\hat{q}(\mathbf{x})$ from the predictive distribution, e.g., $\hat{q}(\mathbf{x})=\hat{y}(\mathbf{x})-1.96 s(\mathbf{x})$ under a normal predictive distribution. The proposed improvement function is $I(\mathbf{x})=\max \left\{0, \hat{q}_{\text {min }}^{(n)}-q(\mathbf{x})\right\}$, where $\hat{q}_{\text {min }}^{(n)}$ is the minimum $\hat{q}(\mathbf{x})$ from $n$ runs so far, and $q(\mathbf{x})=y(\mathbf{x})-1.96 s(\mathbf{x})$ is an unobservable random quantity. Treating $s(\mathbf{x})$ as non-stochastic and assuming $y(\mathbf{x}) \sim N\left(\hat{y}(\mathbf{x}), s^{2}(\mathbf{x})\right)$, the corresponding EI criterion is

$$
E[I(\mathbf{x})]=s(\mathbf{x}) \phi(u)+\left(\hat{q}_{m i n}^{(n)}-\hat{y}(\mathbf{x})+1.96 s(\mathbf{x})\right) \Phi(u)
$$

where $u=\left(\hat{q}_{\min }^{(n)}-\hat{y}(\mathbf{x})+1.96 s(\mathbf{x})\right) / s(\mathbf{x})$. Like the EI criterion in (1), EI in (4) facilitates the trade-off between local and global search. One can easily generalize this EI criterion to $E\left[I^{g}(\mathbf{x})\right]$ (as in Schonlau et al. 1998) or introducing a user specified weight (as in Sóbester et al. 2005).

For complex physical phenomena like climate and tidal power, multiple computer simulators with different computational demands are often available for experimentation. For instance, there are $2 \mathrm{D}$ and $3 \mathrm{D}$ codes for the tidal-power application; the 3D version is a higher-fidelity representation of reality but is much more expensive to run. They can be combined to obtain more informed prediction, and Huang et al. (2006) proposed augmented expected improvement for finding the global minimum of the highest-fidelity process, subject to noise.

\section{Summary}

The essence of these approaches for sequential computer experiments is to formulate the scientific objective through an improvement function. Following some initial runs, the next run is chosen to maximize the expected improvement. In contrast to physical experiments, sequential design is convenient, with the computer handling the logistics of iterating analysis of the data so far, choice of the next run, and making the new run. 
With objectives like optimization and contouring in high-dimensional applications, sequential strategies are efficient in terms of solving the problem with a relatively small number of runs. For these reasons, we expect this area of the design of computer experiments will continue to receive considerable research attention from methodologists and users.

Of course, the usefulness of this strategy depends on having a computer model that provides a satisfactory description of the physical process of interest. Such models have to be checked by

reference to real data from the physical process (Bayarri et al. 2007). However, once a model has been adequately validated it provides an efficient route to achieving the objectives discussed here.

\section{References}

Bayarri, M. J., Berger, J. O., Calder, E. S., Dalbey, K., Lunagomez, S., Patra, A. K., Pitman, E. B., Spiller, E. T. \& Wolpert, R. L. (2009), 'Using statistical and computer models to quantify volcanic hazards', Technometrics 51(4), 402-413.

Bayarri, M. J., Berger, J. O., Paulo, R., Sacks, J., Cafeo, J. A., Cavendish, J., Lin, C. H. \& Tu, J. (2007), 'A framework for validation of computer models', Technometrics 49(2), 138-154.

Bichon, B., Mahadevan, S. \& Eldred, M. (2009), Reliability-based design optimization using efficient global reliability analysis, in '50th AIAA/ASME/ASCE/AHS/ASC structures, structural dynamics, and materials conference.', AIAA, Palm Springs, California, pp. 2009-2261.

Chipman, H., Ranjan, P. \& Wang, W. (2012), 'Sequential design for computer experiments with a flexible bayesian additive model', Canadian Journal of Statistics 40(4), 663-678.

Currin, C., Mitchell, T., Morris, M. \& Ylvisaker, D. (1991), 'Bayesian prediction of deterministic functions, with applications to the design and analysis of computer experiments', 86(416), 953963.

Henkenjohann, N. \& Kunert, J. (2007), 'An efficient sequential optimization approach based on the multivariate expected improvement criterion', Quality Engineering 19(4), 267-280.

Huang, D., Allen, T., Notz, W. \& Miller, R. (2006), 'Sequential kriging optimization using multiplefidelity evaluations', Structural and Multidisciplinary Optimization 32(5), 369-382.

Jones, D. R., Schonlau, M. \& Welch, W. J. (1998), 'Efficient global optimization of expensive black-box functions', J. of Global Optimization 13(4), 455-492.

Lehman, J. S., Santner, T. J. \& Notz, W. I. (2004), 'Designing computer experiments to determine robust control variables', Statistica Sinica 14, 571-590. 
Loeppky, J. L., Sacks, J. \& Welch, W. J. (2009), 'Choosing the sample size of a computer experiment: A practical guide', Technometrics 51(4), 366-376.

Morris, M. D. \& Mitchell, T. J. (1995), 'Exploratory designs for computational experiments', 43(3), 381-402.

O'Hagan, A. (1992), Some Bayesian numerical analysis, in J. M. Bernardo, J. O. Berger, A. P. Dawid \& A. F. M. Smith, eds, 'Bayesian Statistics 4', Oxford University Press, pp. 345-363.

Ponweiser, W., Wagner, T. \& Vincze, M. (2008), Clustered multiple generalized expected improvement: A novel infill sampling criterion for surrogate models, in 'IEEE Congress of Evolutionary Computation', CEC08, Vienna, pp. 3515-3522.

Ranjan, P. (2013), 'Comment: EI criteria for noisy computer simulators', Technometrics 55(1), 2428. Discussion of 'Quantile-Based Optimization of Noisy Computer Experiments with Tunable Precision’ by Picheny, V., Ginsbourger, D., Richet, Y. and Caplin, G. (2013).

Ranjan, P., Bingham, D. \& Michailidis, G. (2008), 'Sequential experiment design for contour estimation from complex computer codes', Technometrics 50(4), 527-541.

Ranjan, P., Haynes, R. \& Karsten, R. (2011), 'A computationally stable approach to gaussian process interpolation of deterministic computer simulation data', Technometrics 53(4), 366-378.

Roy, S. (2008), Sequential-Adaptive Design of Computer Experiments for the Estimation of Percentiles, Ohio State University. Ph.D. Thesis.

Sacks, J., Schiller, S. B. \& Welch, W. J. (1989), 'Designs for computer experiments', Technometrics 31(1), 41-47.

Sacks, J., Welch, W. J., Mitchell, T. J. \& Wynn, H. P. (1989), 'Design and analysis of computer experiments (with discussion)', Statistical Science 4(4), 409-435.

Santner, T. J., Williams, B. J. \& Notz, W. I. (2003), The Design and Analysis of Computer Experiments, Springer, New York.

Schonlau, M., Welch, W. J. \& Jones, D. R. (1998), Global versus local search in constrained optimization of computer models, in N. Flournoy, W. F. Rosenberger \& W. K. Wong, eds, 'New developments and Applications in Experimental Design', Vol. 34, Hayward, CA: Institute of Mathematical Statistics, pp. 11-25.

Sóbester, A., Leary, S. J. \& Keane, A. J. (2005), 'On the design of optimization strategies based on global response surface approximation models', J. of Global Optimization 33(1), 31-59. 